\title{
Size-dependent standard deviation for growth rates: Empirical results and theoretical modeling
}

\author{
Boris Podobnik* \\ Department of Physics, Faculty of Civil Engineering, University of Rijeka, Rijeka, Croatia; \\ Zagreb School of Economics and Management, Zagreb, Croatia; \\ and Center for Polymer Studies and Department of Physics, Boston University, Boston, Massachusetts 02215, USA \\ Davor Horvatic \\ Department of Physics, Faculty of Science, University of Zagreb, Zagreb, Croatia \\ Fabio Pammolli \\ Faculty of Economics, University of Florence and IMT Institute for Advanced Studies, Lucca, Italy \\ Fengzhong Wang and H. Eugene Stanley \\ Center for Polymer Studies and Department of Physics, Boston University, Boston, Massachusetts 02215, USA \\ I. Grosse \\ Martin Luther University, Institute of Computer Science, Halle, Germany \\ (Received 26 September 2007; revised manuscript received 7 January 2008; published 8 May 2008)
}

\begin{abstract}
We study annual logarithmic growth rates $R$ of various economic variables such as exports, imports, and foreign debt. For each of these variables we find that the distributions of $R$ can be approximated by double exponential (Laplace) distributions in the central parts and power-law distributions in the tails. For each of these variables we further find a power-law dependence of the standard deviation $\sigma(R)$ on the average size of the economic variable with a scaling exponent surprisingly close to that found for the gross domestic product (GDP) [Phys. Rev. Lett. 81, 3275 (1998)]. By analyzing annual logarithmic growth rates $R$ of wages of 161 different occupations, we find a power-law dependence of the standard deviation $\sigma(R)$ on the average value of the wages with a scaling exponent $\beta \approx 0.14$ close to those found for the growth of exports, imports, debt, and the growth of the GDP. In contrast to these findings, we observe for payroll data collected from 50 states of the USA that the standard deviation $\sigma(R)$ of the annual logarithmic growth rate $R$ increases monotonically with the average value of payroll. However, also in this case we observe a power-law dependence of $\sigma(R)$ on the average payroll with a scaling exponent $\beta \approx-0.08$. Based on these observations we propose a stochastic process for multiple cross-correlated variables where for each variable (i) the distribution of logarithmic growth rates decays exponentially in the central part, (ii) the distribution of the logarithmic growth rate decays algebraically in the far tails, and (iii) the standard deviation of the logarithmic growth rate depends algebraically on the average size of the stochastic variable.
\end{abstract}

DOI: 10.1103/PhysRevE.77.056102

PACS number(s): 89.65.Gh, 89.65.-s, 82.20.Uv, 02.50.Ey

\section{INTRODUCTION}

The dynamics of noise-driven stochastic systems described by temporal stochastic processes are of interest in a variety of phenomena such as Brownian motion [1], Johnson noise [2], company growth [3], chemical reactions [4], stellar dynamics [5], and quantum optics [6]. In economics, stochastic processes have been successfully applied to model diverse levels of economics systems, ranging from the "micro" level of company products to the "macro" level of company sizes and even national economies. Since the pioneering work of Gibrat [3], researchers have analyzed the relationship between the size of a company and its growth rate [7-14]. For countries, it has been found that the logarithmic growth rates of the gross domestic product (GDP) are approximately (i) double exponentially (Laplace) distributed in the central part $[15,16]$ and (ii) power-law distributed in

*bp@phy.hr the tails [12] with (iii) a power-law relation between the average GDP and the standard deviation of the logarithmic growth rates with a scaling exponent $\beta \approx 0.15$ [15]. These results obtained for macroeconomic data are in agreement with those obtained for microeconomic data, such as sales of different companies [9]. In Ref. [9] the scaling behavior of the growth of U.S. companies was investigated, and the same power-law scaling of the standard deviation of the logarithmic growth rate with the initial size was found. Interestingly, the same power-law scaling behavior was also found for both the sales and the number of employees. Recently, some other microeconomic variables (the number of products of pharmaceutical companies) were analyzed [12], and the same scaling behavior was observed. Analyses of different markets have shown that also the distributions of growth rates of firms, companies, and industrial production can be approximated by tent-shaped exponential distributions $[17,18]$, and exponential-power (Subbotin) distributions [19]. 
TABLE I. First four moments of the distribution of $R$ for exports, imports, and debt. We calculate the annual logarithmic growth rate $R_{t}^{(i)} \equiv \ln \left(S_{t+1}^{(i)} / S_{t}^{(i)}\right)$ for each year $t$ and each country $i$. From the set of all $R_{t}^{(i)}$ we compute the average growth rate $\langle R\rangle$, the median of $R$, the standard deviation $\sigma(R)$, the skewness of $R$, and the kurtosis of $R$. If the distribution of $R$ were Gaussian, the skewness of $R$ would be equal to zero, and the kurtosis of $R$ would be equal to 3 . We find that the skewness of $R$ is greater than zero for all of the three economic variables, stating that in all three cases the distribution of $R$ is asymmetric with a thin tail on the left and a thick tail on the right. We also find that the kurtosis of $R$ is greater than 3 for all of the three economic variables, stating that in all three cases the distribution of $R$ has thicker tails than a Gaussian distribution with the same mean and variance. Interestingly, with respect to both the third and the fourth moment, the deviation from the Gaussian distribution is greatest for debt and smallest for imports.

\begin{tabular}{lccc}
\hline \hline & Export & Import & Debt \\
\hline Years & $1966-2005$ & $1966-2005$ & $1970-2005$ \\
Length & 4049 & 4049 & 3789 \\
No. countries & 174 & 174 & 135 \\
$\langle R\rangle$ & 0.076 & 0.073 & 0.124 \\
Median of $R$ & 0.076 & 0.075 & 0.083 \\
$\sigma(R)$ & 0.184 & 0.169 & 0.251 \\
Skewness of $R$ & 0.314 & 0.198 & 5.665 \\
Kurtosis of $R$ & 11.43 & 7.705 & 71.59 \\
\hline \hline
\end{tabular}

\section{EMPIRICAL ANALYSIS}

We ask if the mechanism found for the time evolution of the country GDP could be responsible for the observed dynamics of three different economic variables: total exports (exports of goods and services), total imports (imports of goods and services), and foreign debt for different countries and different years [20]. For each of these three economic variables $S$, each country $i$, and each year $t$ ranging from 1966 to 2005 [21], we compute the annual logarithmic growth rate as

$$
R_{t}^{(i)} \equiv \ln \left(S_{i+1}^{(i)} / S_{t}^{(i)}\right)
$$

and join all $\left(R_{t}^{(i)}, S_{t}^{(i)}\right)$ pairs into one common data set. Table I summarizes five statistical features of the three analyzed data sets. In order to investigate if and how $\sigma(R)$ correlates with $\langle S\rangle$, we divide the data set into ten subsets by selecting ten subintervals of equal size of $\ln S$.

In Fig. 1 we find that, for each economic variable $S$, the standard deviation $\sigma(R)$ of the annual logarithmic growth rate $R$ decreases algebraically (power law) with the annual average size $\langle S\rangle$ of the economic variable $S$, i.e.,

$$
\sigma(R) \propto\langle S\rangle^{-\beta} .
$$

Surprisingly, the three scaling exponents $\beta$ are close to the scaling exponent $\beta \approx 0.15$ reported for the growth of the GDP [15]. We also investigate the annual growth rate for foreign direct investments, and find it has a similar value of $\beta$.

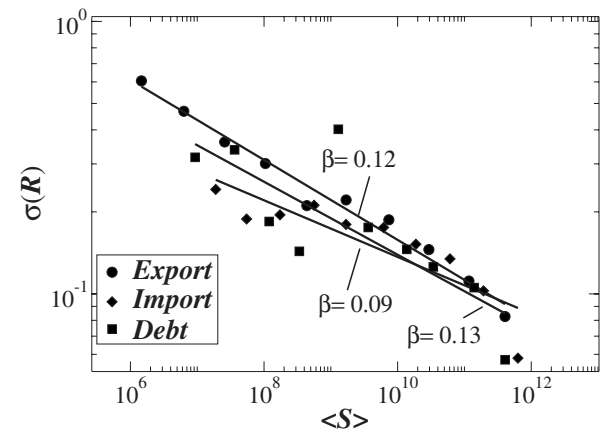

FIG. 1. Standard deviation $\sigma(R)$ of the logarithmic growth rate $R$ as a function of the average value $S$ of imports, exports, and debt. We find that, for each of the three economic variables, the standard deviation $\sigma(R)$ decays algebraically with $S$. Interestingly, all three scaling exponents $\beta$ are similar to each other and surprisingly similar to the scaling exponent $\beta \approx 0.15$ observed for the gross domestic product of countries (GDP) and firms.

Next we investigate how sensitively the values of the scaling exponents depend on the number of subsets chosen. Table II shows that the scaling exponents $\beta$ vary only little with the number of subsets chosen. The results in Ref. [15] imply that the growth rates $R$ from the high GDP countries have a lower standard deviation than the growth rates from the low GDP countries. Surprisingly, we find the same scaling relation for imports, exports, and debt. In order to explain why, for example, debt and GDP exhibit a similar scaling behavior, we note that, from an economic perspective, governments are good if they are capable of maintaining debt in a stable proportion to the GDP.

We stress that the first reason why we join data of all countries is to increase the statistics, since there are at most 40 data points for each country. The second reason is to investigate the global behavior of the economics variables analyzed in the paper. However, it would be interesting to accomplish the data analysis focused on individual countries. Thus, we propose the following procedure. For each country with $n$ values of $R$, we first calculate the average

$$
\langle R\rangle=\frac{1}{n} \sum_{t=1}^{n} R_{t} .
$$

Second, we calculate the scaling exponent $\beta$ for each of the three economic variables, exports, imports, and debt, and for each country, and we obtain that the values of $\beta$ can be both positive and negative, but the average scaling experiment is

TABLE II. Scaling exponents $\beta$ for exports, imports, and debt for different values of the number of subsets. We find that the three scaling exponents are almost independent of the number of subsets.

\begin{tabular}{lccc}
\hline \hline & Export & Import & Debt \\
\hline 7 & $0.12 \pm 0.02$ & $0.08 \pm 0.01$ & $0.13 \pm 0.02$ \\
10 & $0.12 \pm 0.01$ & $0.09 \pm 0.01$ & $0.13 \pm 0.02$ \\
15 & $0.13 \pm 0.01$ & $0.09 \pm 0.01$ & $0.14 \pm 0.02$ \\
20 & $0.11 \pm 0.01$ & $0.09 \pm 0.01$ & $0.14 \pm 0.02$ \\
\hline \hline
\end{tabular}




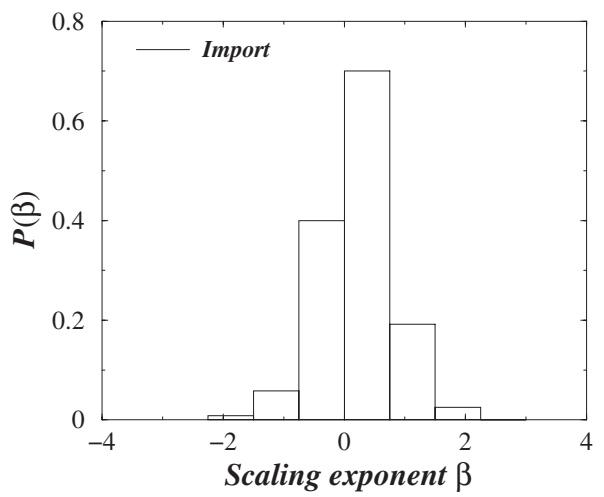

FIG. 2. Probability distribution of the country-specific scaling exponents $\beta$ for import data. For each country, we estimate $\beta$ by a linear regression of $\ln (R-\langle R\rangle)^{2}$ versus $-2 \beta \ln S$. We find that the scaling exponents $\beta$ vary strongly from country to country, ranging from strongly negative values smaller than -2 to strongly positive values greater than +2 . Interestingly, the average scaling exponent $\beta \approx 0.16$ is positive and comparable to the value $\beta \sim 0.09$ obtained from the pooled data set of all countries.

positive for each economic variable. In Fig. 2 we show a probability distribution for import data, and we find that positive scaling exponents prevail giving

$$
\langle\beta\rangle=0.16 \pm 0.74
$$

The same analysis carried out for export data yields

$$
\langle\beta\rangle=0.10 \pm 0.71
$$

and for the debt data we obtain

$$
\langle\beta\rangle=0.16 \pm 0.25 \text {. }
$$

Note that the standard deviations are very high, but the average scaling exponents are close to those obtained in Fig. 1.

Motivated by the results found in Fig. 1, we investigate how the growth-rate distribution depends on the initial size. We partition the data set into three subsets of equal size according to the export value. In Fig. 3, for the subsets with the smallest and the largest values of $S$, we find that (i) the central parts of the empirical conditional distributions of growth rates $R$ are consistent with the Laplace distribution $P(R)=1 /(\sqrt{2} \sigma) \exp [(-\sqrt{2}|R-a|) / \sigma]$. Both parameters a $=$ median of $R$ and $\sigma \equiv \sqrt{2} / N \Sigma\left|R_{t}-a\right|$ are the maximum likelihood estimates of the scalar parameters of the Laplace distribution. We also find that (ii) the spread of the distribution measured by the standard deviation of $R$ decreases with an increase of $S$, consistent with Fig. 1. Again, we find that changing the number of subsets (e.g., from three to four) leaves both findings (i) and (ii) unchanged. Note that by conditional distributions we assume distributions calculated conditional on a specific group of data, not specific initial value $S_{0}$, as commonly defined in probabilistic theory.

Next, we investigate the growth-rate distributions of exports, imports, and debt for all countries and all years. We calculate probabilities by measuring the empirical growth rates at equally spaced growth-rate subintervals. In Figs. 4(a)-4(c) we find that, for all of the three economic variables

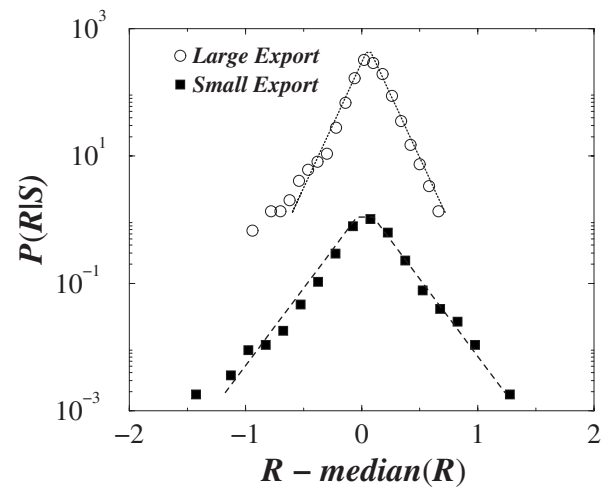

FIG. 3. Conditional probability distributions $P(R \mid S)$ of the logarithmic growth rate $R$ of exports for two values of $S$. We stratify the whole data set $\left(R_{t}^{(i)}, S_{t}^{(i)}\right)$ into three equally large subsets according to the values of $S_{t}^{(i)}$. The lower curve (filled circles) shows the conditional probability distribution $P(R \mid S)$ for the subset with the greatest values $S$ of exports, $S>8.8 \times 10^{9}$, and the upper curve (open circles) shows the conditional probability distribution $P(R \mid S)$ for the subset with the smallest values of exports, $S \leqslant 9.2 \times 10^{8}$. Note that the distributions are shifted for the sake of clarity. We find that the central parts of both of the conditional distributions can be approximated by Laplace distributions centered at the median of $R$. We further find that the standard deviations $\sigma(R)$ of both distributions are significantly different. Specifically, we find that $\sigma(R)$ $\sim 0.139$ for the group with the greatest values $S$ of exports is smaller than $\sigma(R) \sim 0.223$ for the group with the smallest values $S$ of exports, consistent with the observation from Fig. 1 that the standard deviation $\sigma(R)$ decreases with increasing $S$.

$S$, the central parts of the empirical probability distributions $P(R)$ can be approximated by a Laplace distribution, where the median of $R$ is obtained over all countries and all years.

In contrast to the central part, the far tails of the conditional probability distributions cannot be approximated by Laplace distributions. We find that the far right tails $[R$ $\geqslant 0.8$ in Fig. 4(a)] of the conditional probability distributions can be approximated by a power law $P(R) \propto R^{-\alpha}$, consistent with similar findings for both financial $[22,23]$ and economic [12] data.

To estimate the scaling exponent, we employ the equation $\alpha=1+n\left[\sum_{t=1}^{n} \ln \left(R_{t} / R_{\min }\right)\right]^{-1}$ [24], where $R_{\min }$ is the smallest value of $R_{t}$ for which the power-law behavior holds, and the sum runs only over those values of $R_{t}$ that exceed $R_{\min }$. For chosen $R_{\min }=0.8$, for exports, imports, and debt we obtain the following results: $\alpha=4.3, \alpha=5.5$, and $\alpha=3.0$, which are comparable to the scaling exponent $\alpha=4$ obtained for the growth-rate distribution of the country GDP [12].

By analyzing different levels of aggregation of economic systems, from microeconomics to macroeconomics, different research groups have found that many economics variables exhibit a power-law scaling of the standard deviation with its size. Here, we analyze data of countries that report pay data (wages) for each year and all years cumulatively for at least one of the 161 occupations. The number of countries that report pay data for at least one occupation varies between 42 and 76 in the years 1983-2002 [25]. We compute the annual logarithmic growth rate $R_{t}^{(i)} \equiv \ln \left(S_{t+1}^{(i)} / S_{t}^{(i)}\right)$, for each wage $S$ and each country $i$, between two subsequent years $t$ and $t$ 

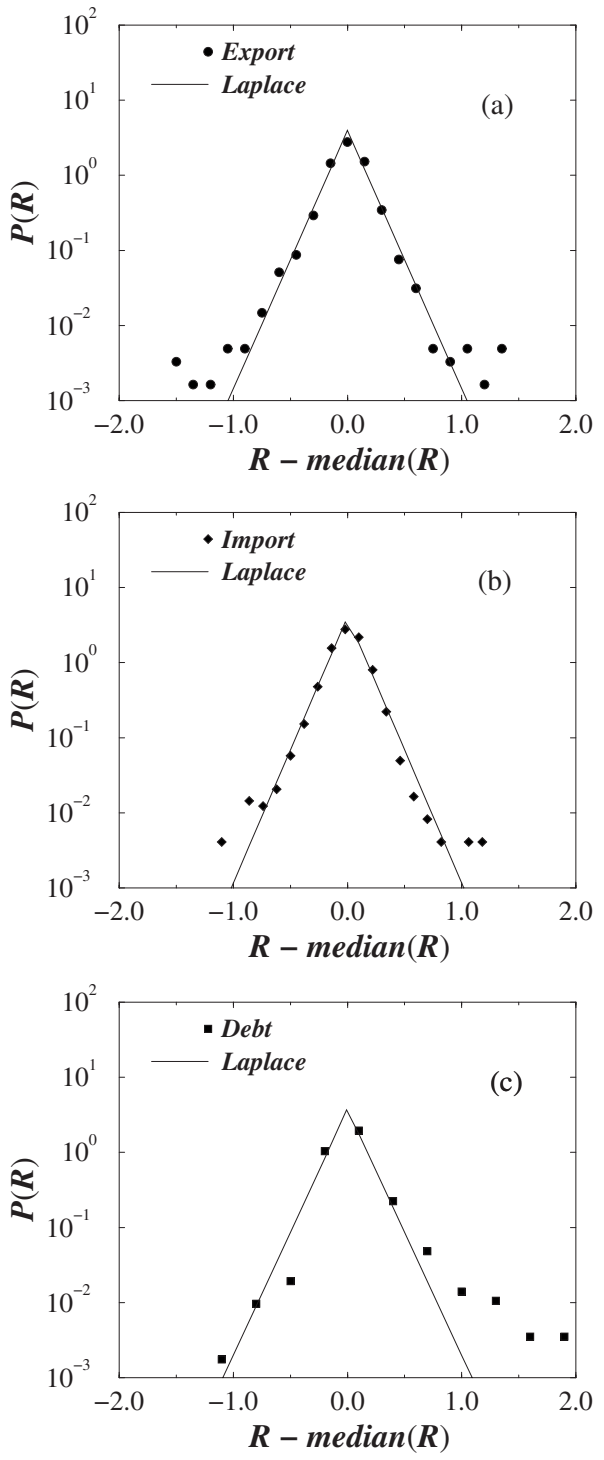

FIG. 4. Probability distributions $P(R)$ of the logarithmic growth rates $R$ of (a) exports, (b) imports, and (c) debt. We find that, for each of the three economic variables, the central parts of $P(R)$ can be approximated by a Laplace distribution centered at the median of $R$, and $N$ denotes the number of data points. Consistent with Table I the standard deviation $\sigma(R)$, the skewness of $R$, and the kurtosis of $R$ are greatest for debt and smallest for imports.

+1 (if they exist). We then join all $\left(R_{t}^{(i)}, S_{t}^{(i)}\right)$ pairs into one common data set. We divide the data set into five equal-size subsets of $\ln S$. Figure 5(a) shows that the standard deviation $\sigma(R)$ of the annual logarithmic growth rate $R$ decreases as a power law with $\langle S\rangle, \sigma(R) \propto\langle S\rangle^{-\beta}$. The scaling exponent

$$
\beta=0.14 \pm 0.04
$$

is close to the scaling exponent $\beta \approx 0.15$ reported for the growth of the GDP [15]. If the data set is divided into six subsets, we obtain a slightly smaller scaling exponent

$$
\beta=0.10 \pm 0.03 \text {. }
$$

In Fig. 5(b) we find that the probability distribution $P(R)$ of the logarithmic growth rates $R$ of wages can be well ap-
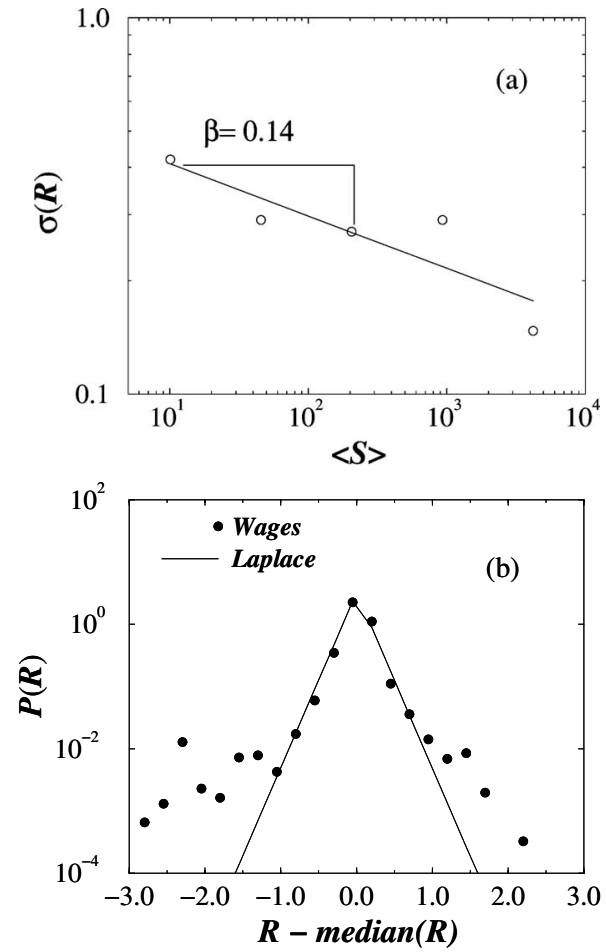

FIG. 5. (a) Standard deviation $\sigma(R)$ of the logarithmic growth rate $R$ as a function of the average value $S$ of wages (expressed in U.S. dollars) of 161 different occupations and different countries. We divide the whole data set $\left(R_{t}^{(i)}, S_{t}^{(i)}\right)$ into five subsets of equal sizes according to the values of $S_{t}^{(i)}$. We find that the standard deviation $\sigma(R)$ decays algebraically with $S$. Interestingly, the scaling exponent $\beta$ is very similar to the scaling exponent $\beta \approx 0.15$ observed for the GDP of countries and firms, and to the scaling exponents $\beta$ found for exports, imports, and debt in Fig. 1. For the average, the median, and the standard deviation of $R$ we obtain $\langle R\rangle=0.042$, median $=0.05$, and $\sigma=0.28$, respectively. (b) Probability distribution $P(R)$ of the logarithmic growth rates $R$ of wages. We find that the central parts of $P(R)$ can be approximated by a Laplace distribution centered at the median of $R$, where the far tails of $P(R)$ can be approximated by a power law $R^{-\alpha}$ with scaling exponent $\alpha \sim 4.2$.

proximated by a Laplace distribution in the central part, while the far right tails can be well approximated by a power law $R^{-\alpha}$ with scaling exponent $\alpha \sim 4.2$.

\section{UNIVARIATE STOCHASTIC PROCESS}

In an attempt to propose a model that could simultaneously reproduce the observed power-law scaling of $\sigma(R)$ with $\langle S\rangle$ and the conditional distribution of $R_{t}$, we propose the following multiplicative discrete-time stochastic process of logarithmic growth rates,

$$
R_{t} \equiv \ln \left(\frac{S_{t}}{S_{t-1}}\right)=\mu_{0} \Delta t+\left(S_{t-1}\right)^{-\gamma} \sigma_{0} \eta_{t} \Delta t,
$$

where $\eta_{t}$ is an independent and identically distributed (i.i.d.) Gaussian noise term with mean $\left\langle\eta_{t}\right\rangle=0$ and variance $\left\langle\eta_{t}^{2}\right\rangle$ $=1$ [26]. Here, the parameter $\mu_{0}$ is related to the expected 


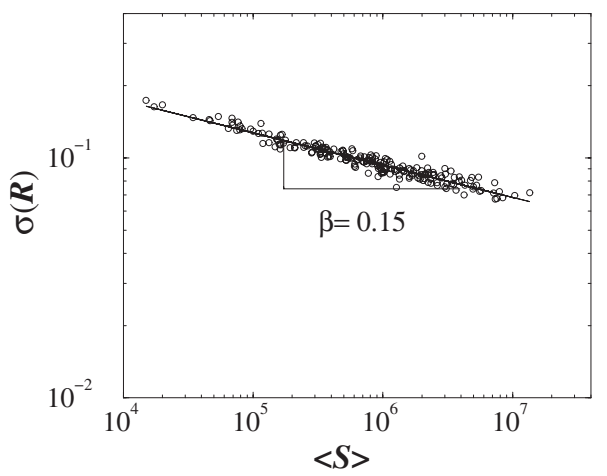

FIG. 6. Dependence of $\sigma(R)$ on $S$ for time series generated by the stochastic process of Eq. (9). We generate 300 times series $S_{t}$, each of the same length $n=200$, by the stochastic process of Eq. (9) with parameter values $\mu_{0}=0.01, \sigma_{0}=0.5, \gamma=0.15$, and $S_{0}=10000$. We compute the average size $\langle S\rangle$ and the standard deviation $\sigma(R)$ from each of the 300 time series, and show a log-log scatter plot of $\sigma(R)$ versus $\langle S\rangle$. We find that the dependence of $\sigma(R)$ on $\langle S\rangle$ can be approximated by a power law, $\sigma(R) \propto\langle S\rangle^{-\beta}$, with scaling exponent $\beta=\gamma$.

growth rate $\langle R\rangle, \sigma_{0}$ controls the spread of $R$, and the scaling parameter $\gamma$ controls the scaling exponent of the power-law dependence of $\sigma(R)$ on $\langle S\rangle$. The dependence of $\sigma(R)$ on $\langle S\rangle$ emerges from the relation $\sigma(R)^{2} \equiv\left\langle\left(R-\mu_{0}\right)^{2}\right\rangle=\sigma_{0}^{2}\left\langle(S)^{-2 \gamma}\right\rangle$, where $\sigma(R)$ decreases with $\langle S\rangle$ for $\gamma>0$.

The stochastic process of Eq. (9) is motivated by the stochastic process of proportional growth rates $[27,28]$

$$
R_{t}^{\prime} \equiv \frac{\Delta S_{i}}{S_{t-1}}=\mu_{0} \Delta t+\left(S_{t-1}\right)^{-\gamma} \sigma_{0} \eta_{t} \Delta t,
$$

where there is virtually no difference between the stochastic processes of Eqs. (9) and (10) in the limit $\left|\Delta S_{t}\right| \ll S_{t-1}$, due to the limit transformation $\ln \left(S_{t} / S_{t-1}\right)=\ln \left[\left(S_{t-1}+\Delta S_{t}\right) / S_{t-1}\right]$ $\approx \Delta S_{t} / S_{t-1}$.

The difference between the stochastic processes of Eqs. (9) and (10) increases with increasing $\Delta S_{t} / S_{t-1}$. The discretetime stochastic process of Eq. (10) leads to unacceptable negative values of $S$ for negative noise terms $\eta_{t}$ (when $R_{t}^{\prime}$ $<-1)$, due to the relation $S_{t}=S_{t-1}\left(1+R_{t}^{\prime}\right)$. In contrast, the stochastic process of Eq. (9) leads to exponentially decreasing, but positive, values $S_{t}=S_{t-1} \exp \left(R_{t}\right)$ for negative values of $R_{t}$. This means that the variable $S$ fluctuates (due to the noise term $\eta$ ) around the exponential trend determined by the first term on the right hand side of Eq. (9).

With the goal of modeling findings (i)-(iii) both qualitatively and quantitatively, we choose $\gamma=0.15$ [9] and generate $N=300$ time series $S_{t}$ all of the same length $n=200$ by the discrete-time stochastic process of Eq. (9). The choice of $S_{0}$ is arbitrary. For each time series $S$, we calculate the logarithmic growth rates $R$, the average size $\langle S\rangle$, and the standard deviation $\sigma(R)$. Figure 6 shows $\sigma(R)$ versus $\langle S\rangle$.

Qualitatively, we find that time series with a small average value of $S$ show a high standard deviation of the annual growth rate $R$, whereas time series with a high average value of $S$ show a small standard deviation of the annual growth rate $R$. Quantitatively, we find that the standard deviation
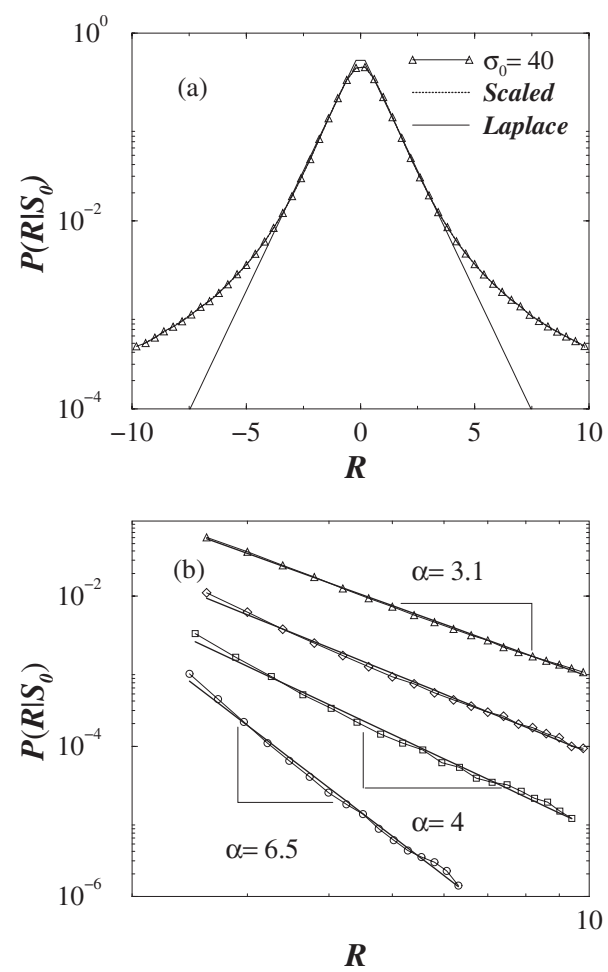

FIG. 7. Probability distribution $P(R)$ for time series generated by the stochastic process of Eq. (9). For four different values of $\sigma_{0}$, we generate $10^{6}$ time series $S_{t}$, each of the same length $n=30$, by the stochastic process of Eq. (9) with parameter values $\mu_{0}=0.01$ and $\gamma=0.15$. Specifically, we choose the four values $\sigma_{0}=40, \sigma_{0}$ $=20, \sigma_{0}=15$, and $\sigma_{0}=10$. For $\sigma_{0}=40$, we choose $S_{0}=10^{10}$, and for $\sigma_{0}^{\prime}=30, \sigma_{0}^{\prime}=15$, and $\sigma_{0}^{\prime}=10$ we choose $S_{0}^{\prime}$ satisfying the relation $\sigma_{0} S_{0}^{\gamma}=\sigma_{0}^{\prime} S_{0}^{\prime \gamma}$. We study the four probability distributions of the resulting $3 \times 10^{7}$ values of $R$ for these four different values of $\sigma_{0}$ and $S_{0}$. (a) We find that the four different probability distributions $P(R)$ collapse after rescaling of $R$, and that the central part of the collapsed probability distribution $P(R)$ can be approximated by a Laplace distribution. (b) We find that the tails of the four probability distributions $P(R)$ can be approximated by a power law $R^{-\alpha}$ with a positive scaling exponent $\alpha$ whose magnitude is monotonically decreasing with an increasing value of $\sigma_{0}$.

$\sigma(R)$ decreases algebraically with the average value of $S$, i.e. $\sigma(R) \propto\langle S\rangle^{-\beta}$, with a scaling exponent $\beta=\gamma$. This states that the univariate stochastic process of Eq. (9) can, qualitatively and quantitatively, reproduce observation (iii).

Next, we investigate if the stochastic process proposed in Eq. (9) could possibly also reproduce observation (i). We generate many time series of length $n=30$, where each time series is obtained with equal $S_{0}$. In Fig. 7(a), for $\gamma=0.15$, we find that the central part of the conditional probability distribution $P\left(R \mid S_{0}\right)$ is more consistent with a Laplace distribution than a Gaussian distribution. This is surprising, because the Laplace distribution is obtained as the superposition of Gaussian distributions of $\eta$ in Eq. (9). So far, the stochastic process proposed in Eq. (9) reproduces observations (i) and (iii).

Further, we investigate if the stochastic process of Eq. (9) could possibly also reproduce observation (ii). In Fig. 7(b), we plot the far tails of $P\left(R \mid S_{0}\right)$ in a log-log plot, and find that 
the tails of $P\left(R \mid S_{0}\right)$ can be approximated by a power law. This is a requiring property because a power law in the far tails is a common behavior for both financial $[22,23]$ and economic [12] data, and commonly modeled by multiplicative stochastic processes [29]. Figure 7(b) shows that by tuning the parameter $\sigma_{0}$, one obtains virtually any scaling exponent of the power-law tails. This property that the tail slopes are inversely proportional to the noise intensity [controlled by the value of $\sigma_{0}$ for the stochastic process of Eq. (9)] is associated with a large class of multiplicative stochastic processes.

Generally, we find that time series of the stochastic process of Eq. (9) with different initial values $S_{0}$ and $S_{0}^{\prime}$ and different parameter values of $\sigma_{0}$ and $\sigma_{0}^{\prime}$ generate the same conditional distribution if $\sigma_{0} S_{0}^{-\gamma}=\sigma_{0}^{\prime} S_{0}^{\prime-\gamma}$ [Fig. 7(a)]. We conclude that the three empirical findings (i)-(iii) are reproduced by the stochastic process proposed in Eq. (9).

We find that the stochastic process of Eq. (9) can be used for modeling both increasing (with $\gamma<0$ ) [30] and decreasing $(\gamma>0)$ power-law scaling of the standard deviation $\sigma(R)$ with $S$. To this end, we analyze payroll data, denoted by $S$, calculated for each of the 50 states of the USA [31]. We compute for each state $i$, and each year $t$ ranging from 1992 to 2004, the one-year logarithmic growth rate of Eq. (9), $R_{t}^{i}$, and join all $\left(R_{t}^{(i)}, S_{t}^{(i)}\right)$ pairs into one common data set.

Figure 8(a) shows the standard deviation $\sigma(R)$ of the logarithmic growth rates $R$ of total payroll as a function of the average size $\langle S\rangle$. For total payroll we find a power-law scaling $\sigma(R) \propto\langle S\rangle^{-\beta}$ of the standard deviation $\sigma(R)$ with $\langle S\rangle$, in agreement with what was earlier found for countries and companies. However, in contrast to what was found for countries and companies, we find for total payroll that the scaling exponent

$$
\beta=-0.08 \pm 0.03
$$

is negative. With an arbitrarily chosen negative value of $\gamma$, Fig. 8(b) shows that the increasing functional dependence of $\sigma(R)$ on $\langle S\rangle$ can be modeled by the stochastic process of Eq. (9).

To exemplify the utility of the stochastic process of Eq. (9), next we propose a simple model for the growth of business companies. We assume that each company $\alpha$ is comprised of $K_{\alpha}$ units, such as divisions or products. To make the model simple, we assume that neither the number of companies nor the number of company units change in time. At time $t$, each company unit has size $s_{i, t}$, where $i$ $=1,2, \ldots, K_{\alpha}$. We assume $s_{i, t}$ are independent random variables, and we propose that the size $s_{i, t}$ of each company unit is governed by the stochastic process of Eq. (9), where the initial sizes $s_{i, 0}$ are drawn from a Gaussian distribution. The size of a company is defined as $S_{\alpha, t} \equiv \sum_{i=1}^{K_{\alpha}} s_{i, t}$. The growth rate of each company is defined as $R_{t} \equiv \ln \left(S_{\alpha, t+1} / S_{\alpha, t}\right)$. Finally, for each company we generate a time series $S_{\alpha, t}$ of the same length $n=200$. For each time series $S_{\alpha, t}$, we calculate the logarithmic growth rates $R_{t}$, the average size $\langle S\rangle$, and the standard deviation $\sigma(R)$. Figure 9 shows $\sigma(R)$ versus $\langle S\rangle$, where the power-law dependence is consistent with the empirically found power-law relationship between firm size and growth-rate standard deviation [9].
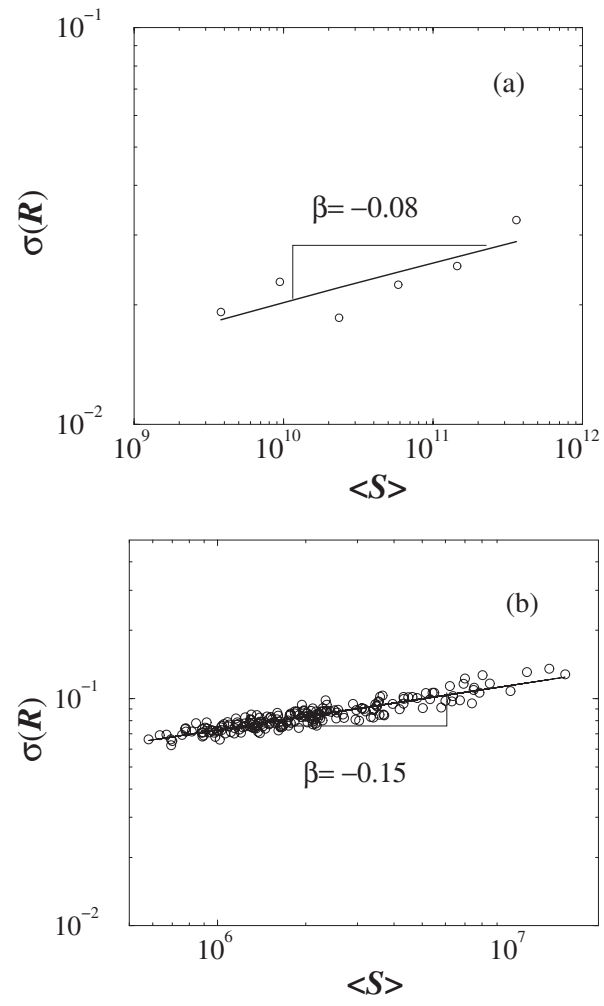

FIG. 8. Standard deviation $\sigma(R)$ of the annual logarithmic growth rate $R$ as a function of the average size $\langle S\rangle$ of total payroll expressed in U.S. dollars. In contrast to the observation found for countries (Fig. 1) and wages (Fig. 2), we find that for total payroll the standard deviation $\sigma(R)$ increases with $S$. However, in agreement with the observations of Figs. 1 and 2, the dependence of $\sigma(R)$ on $S$ can be approximated by a power law, with a negative scaling exponent $(\beta=-0.08 \pm 0.03)$. For the average and the median of $R$ we obtain $\langle R\rangle=0.054$ and median of $R=0.057$, respectively. (b) Standard deviation $\sigma(R)$ as a function of $S$ for time series generated by the stochastic process of Eq. (9). We generate 300 time series $S_{t}$, each of the same length $n=200$, by the stochastic process of Eq. (9) with parameter values $\mu_{0}=0.02, \sigma_{0}=0.01, \gamma=-0.15$, and $S_{0}=10^{5}$. We find qualitatively that for negative values of $\gamma$ the standard deviation $\sigma(R)$ grows monotonically with $S$ and quantitatively that the dependence of $\sigma(R)$ on $S$ can be approximated by a power law $S^{-\beta}$ with a negative scaling exponent $\beta \approx \gamma$.

In conclusion, we find that, in contrast to the stochastic process of Eq. (10), the univariate stochastic process of Eq. (9) can reproduce, both qualitatively and quantitatively, findings (i)-(iii) that are typical for a wide range of financial and economic variables.

\section{MULTIVARIATE STOCHASTIC PROCESS}

Financial and economic variables, such as exports, imports, debt, and the GDP, are often cross correlated. Hence, we attempt in the following to generalize the univariate stochastic process of Eq. (9) to multiple (cross-correlated) variables. As an example of correlated variables, we expose the time series of exports and imports of Germany in Fig. 10, because export and import data are characterized by cross correlations between their time series [32]. Correlations be- 


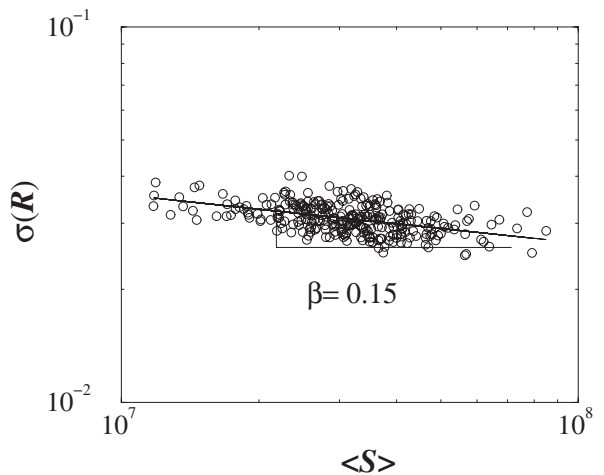

FIG. 9. Standard deviation $\sigma(R)$ of the growth rate $R$ as a function of the average size $\langle S\rangle$ obtained for modeling business companies, denoted by $\alpha$, where $R=\ln \left(S_{\alpha, t} / S_{\alpha, t-1}\right)$ defines the growth rate of each company, and $S_{\alpha, t}$ denotes the size of a company. Each company is comprised of $K_{\alpha}$ units where each company unit has size $s_{i, t}$, we assume $s_{i, t}$ are independent random variables, and it holds $S_{\alpha, t}=\sum_{i=1}^{K} s_{i, t}$. The size of each company unit is governed by the stochastic process of Eq. (9) with parameter values $\mu_{0}=0.02$, $\sigma_{0}=0.7$, and $\gamma=0.15$. We draw initial sizes $s_{i, 0}$ from a Gaussian distribution with a mean of $10^{5}$ and standard deviation of $10^{4}$. We consider 300 different companies, and for each company we generate a time series, each of the same length $n=200$. For each company we calculate the average size $\langle S\rangle$ and the standard deviation $\sigma(R)$. We find that the standard deviation $\sigma(R)$ decreases with $S$ according to a power law with scaling exponent $\beta \approx \gamma$.

tween these two time series are obvious because an increase in exports is practically always followed by an increase in imports. In economics, the GDP for any country is defined as the sum of five economic variables, among which are exports and imports.

In order to test if it is possible to model simultaneously the growth of the GDP and its cross-correlated constituents, all specified by a size dependence of $\sigma(R)$, we define a "theoretical" GDP as the sum of two cross-correlated variables $I_{1}$ and $I_{2}$ [33]. We propose that the logarithmic growth rates for $I_{1, t}$ and $I_{2, t}$ follow a stochastic process that is an extension of the stochastic process of Eq. (9),

$$
R_{I_{1}, t} \equiv \ln \left(\frac{I_{1, t}}{I_{1, t-1}}\right)=\mu_{0}+\sigma_{0} I_{1, t-1}^{-\gamma}\left(\eta_{I_{1}, t}+\phi R_{I_{2}, t-1}\right),
$$

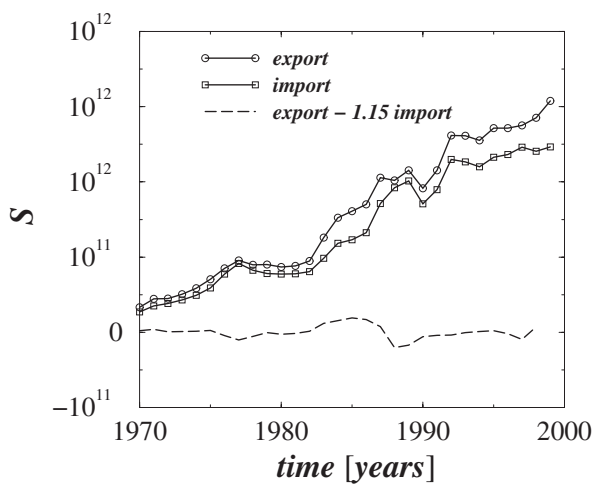

FIG. 10. Time series of exports and imports of Germany expressed in U.S. dollars. We find that exports and imports are highly correlated.

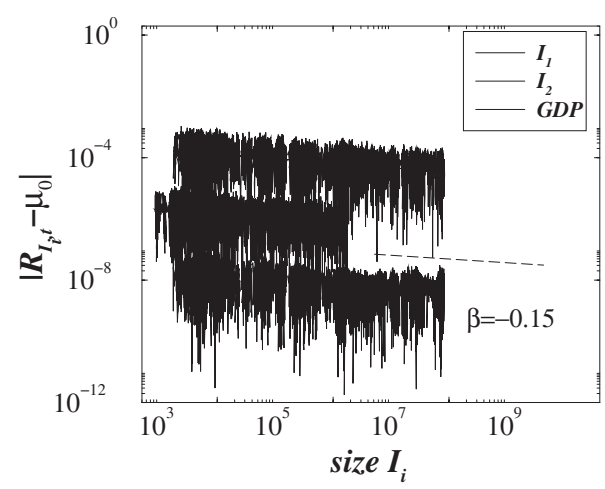

FIG. 11. Dependence of the magnitude $\left|R_{I_{i}, t}-\mu_{0}\right|$ of the logarithmic growth rate $R_{I_{i}, t}$ on the value of $I_{i}$ in the presence of cross correlations. We generate a bivariate time series $I_{t}^{(1)}, I_{t}^{(2)}$ of length $n=10000$ by the stochastic process of Eqs. (11) and (12) with parameter values $\mu_{0}^{(1)}=0.002, \mu_{0}^{(2)}=0.001, \sigma_{0}^{(1)}=\sigma_{0}^{(2)}=0.1, \gamma^{(1)}=\gamma^{(2)}$ $=0.15, \phi=0.9, I_{1,0}=2000$, and $I_{2,0}=3000$. We find that, for all $i$ $=1,2,3,\left|R_{I_{i}, t}-\mu_{0}^{(i)}\right|$ is monotonically decreasing with $I_{i}, t$ and that the dependence of $\left|R_{I_{i}, t}-\mu_{0}\right|$ on $I_{i}, t$ can be approximated by a power law with a scaling exponent $\beta \approx \gamma$.

$$
R_{I_{2}, t} \equiv \ln \left(\frac{I_{2, t}}{I_{2, t-1}}\right)=\mu_{0}+\sigma_{0} I_{2, t-1}^{-\gamma}\left(\eta_{I_{2}, t}+\phi R_{I_{1}, t-1}\right),
$$

where parameter $\phi$ controls the cross correlations between $I_{1, t}$ and $I_{2, t}$. Note that the process generating the growth of $I_{2, t}$ is similar to the stochastic process generating the growth of $I_{1, t}$, where $I_{1, t}$ and $I_{2, t}$ are exchanged. For simplicity, both stochastic processes generating $I_{1, t}$ and $I_{2, t}$ are defined by equal values for $\mu_{0}$ and $\sigma_{0}$.

For each time step, we perform simulations to calculate two variables, $I_{1, t}$ of Eq. (11) and $I_{2, t}$ of Eq. (12), for different initial values $I_{1,0}$ and $I_{2,0}$, and we compute $(\mathrm{GDP})_{t} \equiv I_{1, t}$ $+I_{2, t}$. In Fig. 11 we find that, regardless of the presence of cross correlations between $I_{1, t}$ and $I_{2, t}$, the magnitude of $R_{I_{i}, t}$ $(i=1,2)$ scales with size as a power law $\left|R_{I_{i}, t}-\mu_{0}\right| \propto\left\langle I_{i, t}\right\rangle^{-i}$ for each of the two variables $I_{i, t}$.

We further find that, not only for each variable $I_{i, t}$, but also for the GDP, the magnitude of $R_{\mathrm{GDP}, t}$ scales with size according to the same power law. We find that these findings are not restricted to the particular parameter value $\gamma=0.15$ consistent with observation (iii). We also find that the standard deviation scales with size as a power law for each variable $I_{1, t}, I_{2, t}$, and GDP independently of the values of $\gamma$.

Several stochastic processes have been proposed for modeling the growth dynamics of complex organization, such as companies [3,7-9,12,16,17,34-39]. However, the dynamics of the proposed stochastic processes are not fully consistent with the three observations (i)-(iii), widely found in empirical data $[9,12]$. For example, the model of $\mathrm{Fu}$ et al. [12] can reproduce findings (i) and (ii), but fails to explain (iii). Second, none of the existing models explains, in the case of different (iv) cross-correlated variables, the size dependence of the standard deviation of growth rates for each of the variables.

\section{CONCLUSIONS}

We find that many economic variables $S$-including exports, imports, and foreign debt-exhibit three ubiquitous 
properties: their logarithmic growth rates $R$ are (i) Laplace distributed in the central part and (ii) power-law distributed in the far tails, and (iii) the standard deviation $\sigma(R)$ decays algebraically with the average size $\langle S\rangle$ with a scaling exponent $\beta \approx 0.15$ surprisingly similar for all three economic variables and surprisingly close to the scaling exponent observed for the GDP.

When analyzing wages for 161 different occupations and total payroll data obtained from 50 of states of the USA, we also find a power-law dependence of $\sigma(R)$ on $\langle S\rangle$ in both cases. However, for the wages data $\sigma(R)$ decreases monotonically with $\langle S\rangle$ with a positive scaling exponent $\beta$ close to that observed for exports, imports, debt, and the GDP, whereas for the payroll data $\sigma(R)$ increases monotonically with $\langle S\rangle$ with a negative scaling exponent $\beta \approx-0.08$.

We propose a univariate stochastic process with only two control parameters that is capable of reproducing, qualita- tively and quantitatively, these three findings. We further show that the parameter $\gamma$ controls the scaling exponent of (iii), and $\sigma_{0}$ controls the scaling exponent of (ii). We propose a multivariate stochastic process that extends the univariate stochastic process to multiple cross-correlated variables, and we find that this stochastic process reproduces each of the three findings (i), (ii), and (iii) for each of the variables. Moreover, we find that the "theoretical" GDP, defined as the sum of the cross-correlated variables, reproduces all three findings (i), (ii), and (iii).

\section{ACKNOWLEDGMENTS}

We thank X. Gabaix and H. Larralde for helpful discussions, and MZOS and NSF for financial support.
[1] A. Einstein, Ann. Phys. 17, 549 (1905).

[2] J. Johnson, Phys. Rev. 32, 97 (1928).

[3] R. Gibrat, Les Inegalites Economiques (Sirey, Paris, 1933).

[4] H. A. Kramers, Physica (Amsterdam) 7, 284 (1940).

[5] S. Chandrasekhar, Rev. Mod. Phys. 15, 1 (1943).

[6] H. Risken, Progress in Optics (North-Holland, Amsterdam, 1970), Vol. 8.

[7] M. Kalecki, Econometrica 13, 161 (1945).

[8] B. Jovanovic, Econometrica 50, 649 (1982).

[9] M. H. R. Stanley et al., Nature (London) 379, 804 (1996); L. A. N. Amaral et al., J. Phys. I 7, 621 (1997); S. V. Buldyrev et al., ibid. 7, 635 (1997).

[10] R. L. Axtell, Science 293, 1818 (2001).

[11] G. Bottazzi and A. Secchi, Econ. Lett. 80, 415 (2003).

[12] D. Fu et al., Proc. Natl. Acad. Sci. U.S.A. 102, 18801 (2005). Relevant extensions of this paper include: J. Growiec et al., Econ. Lett. 98, 207 (2008); S. V. Buldyrev et al., J. Eur. Econ. Assoc. 5, 574 (2007); Eur. Phys. J. B 57, 131 (2007); F. Pammolli et al., ibid. 57, 127 (2007).

[13] A. Palestrini, Econ. Lett. 94, 367 (2007).

[14] H. M. Gupta et al., Physica A 375, 643 (2007).

[15] Y. Lee et al., Phys. Rev. Lett. 81, 3275 (1998).

[16] L. A. N. Amaral et al., Phys. Rev. Lett. 80, 1385 (1998).

[17] A. Ishikawa, Physica A 383, 79 (2007).

[18] F. Cecconi et al., Phys. Rev. Lett. 89, 088102 (2002).

[19] G. Fagiolo et al., Eur. Phys. J. B 57, 205 (2007).

[20] Data available at http://earthtrends.wri.org

[21] For debt data ranges from 1970 to 2005.
[22] T. Lux, Appl. Financ. Econ. 6, 463 (1996).

[23] P. Gopikrishnan et al., Eur. Phys. J. B 3, 139 (1998); Phys. Rev. E 60, 5305 (1999); V. Plerou et al., ibid. 60, 6519 (1999).

[24] M. E. J. Newman, Contemp. Phys. 46, 323 (2005).

[25] Data available at http://www.census.gov

[26] For simplicity, in Eq. (1) we set $\Delta t=1$.

[27] J. C. Cox and S. A. Ross, J. Financ. Econ. 3, 145 (1976).

[28] By using the transformation $y=S^{-\gamma}$, one may show that the continuous-time version of the stochastic process of Eq. (10), $d S(t) / d t=\mu_{0} S(t)+\sigma_{0} S(t)^{1 ! \gamma} \eta(t)$, reduces to the OrnsteinUhlenbeck (OU) process $d y / d t=+\gamma \mu_{0} y+\gamma \sigma_{0} \eta(t)$ defined in physics [G. E. Uhlenbeck and L. S. Ornstein, Phys. Rev. 36, 823 (1930)].

[29] T. Bollerslev, J. Econometr. 31, 307 (1986).

[30] I. M. Janosi and J. A. C. Gallas, Physica A 271, 448 (1999).

[31] Data available at http://www.nber.org/oww/

[32] A. C. Arize, Int. Rev. Econ. Fin. 11, 101 (2002).

[33] For the case of simplicity, we choose only two instead of five variables.

[34] Y. Ijiri and H. A. Simon, Skew Distributions and the Sizes of Business Firms (North-Holland, Amsterdam, 1977).

[35] J. Sutton, J. Econom. Lit. 35, 40 (1997).

[36] H. Takayasu and K. Okuyama, Fractals 6, 67 (1998).

[37] J. Sutton, Physica A 312, 577 (2002).

[38] M. Wyart and J. P. Bouchaud, Physica A 326, 241 (2003).

[39] D. Fu et al., Phys. Rev. E 74, 036118 (2006). 